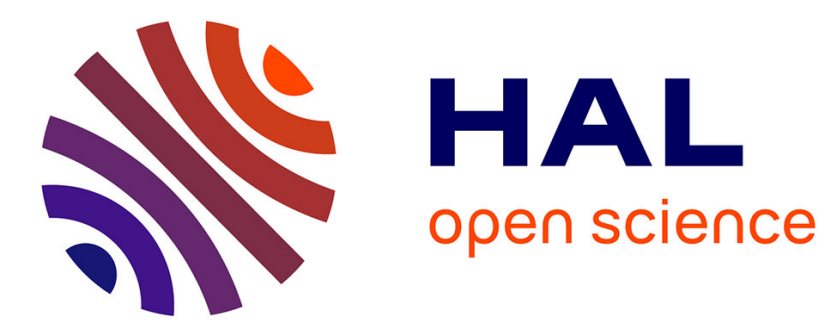

\title{
Static scattering from cyclic copolymers in solution
}

\author{
M. Benmouna, R. Borsali, H. Benoît
}

\section{To cite this version:}

M. Benmouna, R. Borsali, H. Benoît. Static scattering from cyclic copolymers in solution. Journal de Physique II, 1993, 3 (7), pp.1041-1047. 10.1051/jp2:1993180 . jpa-00247881

\section{HAL Id: jpa-00247881 https://hal.science/jpa-00247881}

Submitted on 1 Jan 1993

HAL is a multi-disciplinary open access archive for the deposit and dissemination of scientific research documents, whether they are published or not. The documents may come from teaching and research institutions in France or abroad, or from public or private research centers.
L'archive ouverte pluridisciplinaire HAL, est destinée au dépôt et à la diffusion de documents scientifiques de niveau recherche, publiés ou non, émanant des établissements d'enseignement et de recherche français ou étrangers, des laboratoires publics ou privés. 
Classification

Physics Abstracts

$36.20-61.25 \mathrm{H}-5.70$

\title{
Static scattering from cyclic copolymers in solution
}

\author{
M. Benmouna $(1, * *)$, R. Borsali $(1, *)$ and H. Benoît $\left({ }^{2}\right)$ \\ (') CERMAV-CNRS and University Joseph Fourier, P.O. Box 53x, 38041 Grenoble Cedex, \\ France \\ (2) Institut Charles Sadron, 6 rue Boussingault, 67083 Strasbourg Cedex, France \\ (Received 7 November 1992, revised 29 January 1993, accepted 7 April 1993)
}

\begin{abstract}
Résumé. - Dans cet article, nous étudions les propriétés statiques de diffusion des copolymères cycliques dans une solution satisfaisant la condition théta optique. Le cas d'un dibloc symétrique est examiné en détail. Les résultats montrent des différences importantes par rapport au cas d'un copolymère linéaire. L'interaction entropique qui apparaît dans une chaîne cyclique induit une diminution de l'intensité diffusée et un déplacement de son maximum vers une valeur de $q$ plus élevée. Ces résultats montrent un effet compatibilisant substantiel quand on les compare avec ceux d'un copolymère linéaire.
\end{abstract}

\begin{abstract}
The static scattering properties of cyclic diblock copolymers in solution are studied. The case of a symmetric diblock in a solvent satisfying the optical theta condition is considered in details. The results show substantial differences with respect to linear diblock copolymers. The additional entropic interaction in cyclic chains induces a decrease of the scattering intensity and a shift of the location of its maximum to a higher $q$ value. This results in a substantial compatibility enhancement as compared to linear copolymers.
\end{abstract}

\section{Introduction.}

Cyclic polymers such as the DNA ring [1] are important because of their potential applications and their role in the organization of living cells. They are also of fundamental interest because they provide a simple macromolecular model that can help in elucidating the basic concepts of the physical properties of macromolecular compounds. However, some special behaviors are found in cyclic chains as compared to their linear counterparts. In terms of their thermodynamic properties for example, measurements on dilute solutions of polystyrene in cyclohexane at $35{ }^{\circ} \mathrm{C}$ which is the theta temperature for linear chains, have revealed that the second virial coefficient is positive for cyclic polymers [2-4]. This means that the theta temperature is

(*) To whom correspondence should be addressed.

(**) Permanent Address: University of Tlemcen, Physics Department P.O. Box 119 Tlemcen, Algeria. 
shifted to lower values and that the excluded volume interaction is more significant than for linear chains. Moreover, since the monomer density is higher inside a closed chain as compared to a linear chain, one may expect to see some similarity with branched polymers. Indeed, experiments reported by the Strasbourg group [5] on star molecules have also shown that the theta temperatures for both vanishing second virial coefficient and unperturbed dimensions are below the theta temperature measured for the corresponding linear chains. The structural properties also show some differences essentially because of the end effects which are irrelevant when the two chain ends are chemically linked together. It has been shown using static neutron scattering [4] in dilute solutions of ring polystyrene in cyclohexane that the form factor can be represented by the so-called Cassasa's function $P_{r}(q)$ [6]:

$$
P_{\mathrm{r}}(q)=\frac{2}{\sqrt{u}} \mathrm{e}^{-(u / 4)} \int_{0}^{\sqrt{w / 2}} \mathrm{~d} x \mathrm{e}^{x^{-}}
$$

$u=q^{2} N \sigma^{2} / 6$, where $N$ is the degree of polymerization, $\sigma$ the length of the statistical segment, and $q$ the scattering wavevector $q=(4 \pi / \lambda) \sin (\theta / 2), \lambda$ is the wavelength of the incident radiation and $\theta$, the scattering angle. Neutron scattering experiments made in dilute solutions of linear chains under the same conditions reveal that the form factor satisfies the known Debye function:

$$
P_{\mathfrak{l}}(q)=\frac{2}{u^{2}}\left(\mathrm{e}^{-u}+u-1\right)
$$

The radii of gyration extracted from these data are found to satisfy the relationship

$$
R_{\mathrm{g} \ell}=\sqrt{2} R_{\mathrm{gr}}
$$

where the subscripts $\ell$ and $\mathrm{r}$ refer to linear and ring polymers, respectively. Furthermore, the theory of linear diblock copolymers in the melt has been put forward by Leibler [7] in the framework of the random phase approximation (RPA) [8]. The scattered intensity $I(q)$ in the case of a diblock copolymer is found as follows:

$$
\frac{(a-b)^{2}}{I(q)}=\frac{P_{\ell}(q)}{N f^{2}(1-f)^{2}\left[P_{\ell 1}(q) P_{\ell 2}(q)-P_{\ell 12}^{2}(q)\right]}-2 \chi
$$

$a$ and $b$ represent the scattering lengths of both species if the neutron scattering technique is used, $(a-b)$ is the increment of the refractive index in the case of light scattering. The total form factor of the chain $P(q)$ can be expressed in terms of its usual internal form factors $P_{p_{1}}(q), P_{\ell_{2}}(q)$, and $P_{\ell_{12}}(q)$ by the geometrical relationship [9] :

$$
P_{p}(q)=f^{2} P_{p_{1}}(q)+(1-f)^{2} P_{\ell_{2}}(q)+2 f(1-f) P_{\ell_{12}}(q) .
$$

The subscripts 1 and 2 refer to the blocks $\mathrm{A}$ and $\mathrm{B}$ respectively; $f$ is the composition of monomer A per chain. In the case of a symmetric diblock copolymer, $f=1 / 2$, $P_{\ell_{1}}(q)=P_{\ell_{2}}(q)=P_{\ell_{1 / 2}}(q), 2 P_{\ell}(q)=P_{\ell_{1 / 2}}(q)+P_{P_{12}}(q)$, and equation (4) reduces to:

$$
\frac{(a-b)^{2}}{I(q)}=\frac{4}{N\left[P_{\ell ! / 2}(q)-P_{\ell}(q)\right]}-2 \chi .
$$

The plot of $I(q)$ versus $q$ shows a maximum whose position is located approximately at $q_{\mathrm{m} \ell}=2 / R_{\mathrm{g} \ell}$. The height of this maximum depends on the value of the Flory [10] interaction parameter $\chi$. When this parameter reaches a critical value $\chi_{\mathrm{m}} \ell$ the system undergoes a 
microphase separation transition (MST). Within the RPA, the critical parameter $\chi_{m}$ is found as :

$$
\chi_{\mathrm{m} \ell}=\frac{2}{\left[N\left(P_{\mathrm{m} \ell 1 / 2}-P_{\mathrm{m} \ell}\right)\right]} \cong \frac{10}{N}
$$

where the subscript $\mathrm{m}$ indicates the value of the form factor at $q=q_{\mathrm{m}}$. The purpose of this article is to see whether similar observations can be made for cyclic diblock copolymers. In addition, we introduce a third component which is a low molecular weight solvent and investigate the effects of polymer concentration in the vicinity of the overlap threshold $c^{*}$ and above. This allows for the extension of the RPA to solution properties assuming that the effects of fluctuations can be ignored.

\section{The cyclic copolymer in solution.}

The static scattering properties of linear diblock copolymers in solution were derived within the framework of the RPA [11] and the results were found to be in reasonable agreement with the neutron scattering data $[11,12]$. The general formalism is already known and there is no need to reproduce it here. However, the case of cyclic copolymers has received only limited attention in the literature. To our knowledge, the study of the scattering properties during transesterification reactions by Benoit et al. [13] is the only place where the case of cyclic copolymers is invoked. Here, we show how the results for linear diblocks can be extended to closed chains. For simplicity, we consider the case of a symmetric Gaussian diblock for which the scattering intensity $I(q)$ is given by [12]

$$
\frac{I(q)}{\Phi N}=\left(\frac{a-b}{2}\right)^{2} \frac{\left[P_{\mathrm{r} 1 / 2}(q)-P_{\mathrm{r}}(q)\right]}{1-\frac{\chi}{2} \Phi N\left[P_{\mathrm{r} 1 / 2}(q)-P_{\mathrm{r}}(q)\right]}+\left(\frac{a+b}{2}-s\right)^{2} \frac{P_{\mathrm{r}}(q)}{1+\left(v+\frac{\chi}{2}\right) \Phi N P_{\mathrm{r}}(q)}
$$

where the excluded volume parameter $v$ can be expressed in terms of the polymer-solvent interaction parameter $\chi_{0}$ and the volume fraction of solvent $\Phi_{\mathrm{s}}=1-\Phi$ as follows :

$$
v=\frac{1}{\Phi_{s}}-2 \chi_{0}
$$

For simplicity we have disregarded a proportionality constant which depends on the molar volumes of the three components assuming that they are equal to the volume of a unit cell in the Flory-Huggins [10] lattice model. These numerical refinements can be easily introduced whenever needed. The volume fraction of polymer $\Phi$ should be set to 1 in the bulk limit when no solvent is present. In this limit, $v \rightarrow \infty$ and hence only the first term in the right hand side (RHS) of equation (8) remains and gives a known result in the case of linear diblock copolymer chains [7]. The second term in the RHS of equation (8) describes the scattered intensity one would obtain from a cyclic homopolymer having a form factor $P_{\mathrm{r}}(q)$, a degree of polymerization $N$, and a slightly enhanced excluded volume interaction because of the chemical mismatch of the two polymers (i.e. $v+\chi / 2$ ). The essential difference between these results and those obtained for linear chains is inherent in the form factors $P_{\mathrm{r}}(q)$ and $P_{\mathrm{r} l / 2}(q)$. These quantities are sensitive to the chain architecture and reflect the entropic interactions of its monomers. The main assumption made in their calculation is to write the average square distance between two monomers $i$ and $j$ separated by the chemical distance 
$n=|i-j|$ as $[14]:$

$$
\left\langle r_{n}^{2}\right\rangle=n\left(1-\frac{n}{N}\right) \sigma^{2}
$$

where the symbol $\langle\ldots\rangle$ denotes the thermal average. The second term in the RHS of this equation is due to the fact that one can go from $i$ to $j$ monomers following two different paths along the chain. Using this assumption in the definition of the total form factor, one obtains :

$$
P_{\mathrm{r}}(q)=\int_{0}^{1} \mathrm{~d} x \mathrm{e}^{-u(1-s)}
$$

which yields the Cassasa function in equation (1). The calculation of $P_{\mathrm{r} 1 / 2}(q)$ can be made in a similar way but the $n$ values should be limited to the range from 1 to $N / 2$. One obtains the following simple result :

$$
P_{\mathrm{r} 1 / 2}(q)=\frac{\left[1-\mathrm{e}^{-(u / 4)}\right]}{(u / 4)}
$$

An interesting property of equation (8) is that the two terms carry physical meanings of different natures. It shows that the scattering due to the local composition (or the concentration difference) fluctuations is completely decoupled from the scattering due to the total concentration fluctuations of the polymer in the solvent. Furthermore, one can have direct access to each of these terms by choosing properly the scattering lengths $a, b$, and $s$ or equivalently the indices of refraction depending on the type of radiation to be used. Obviously, the composition fluctuations are more relevant and they can be evaluated unambigously in the so-called zero average contrast condition [12] or equivalently for symmetrical mixtures, in the optical theta condition [14]. This means that :

$$
\frac{a+b}{2}=s
$$

and from equation (8)

$$
\frac{I(q)}{\Phi N}=\left(\frac{a-b}{2}\right)^{2} \frac{\left[P_{\mathrm{r} l / 2}(q)-P_{\mathrm{r}}(q)\right]}{1-\frac{\chi}{2} \Phi N\left[P_{\mathrm{r} 1 / 2}(q)-P_{\mathrm{r}}(q)\right]}
$$

First, one notes that the structure factor multiplying $\left(\frac{a-b}{2}\right)^{2}$ in this equation is proportional to $S_{\ell}(q)$ which is defined by :

$$
S_{\mathcal{P}}(q)=\frac{S_{a a}(q)}{f^{2}}+\frac{S_{b b}(q)}{(1-f)^{2}}-\frac{2 S_{a b}(q)}{f(1-f)}
$$

where $S_{a a}(q), S_{b b}(q)$, and $S_{a b}(q)$ are the partial structure factors due to interferences between $\mathrm{AA}, \mathrm{BB}$, and $\mathrm{AB}$ molecules. In the symmetrical case, one has [12]:

$$
\begin{gathered}
S_{a a}(q)=S_{b b}(q)=S(q)=\frac{P_{\mathrm{r} 1 / 2}(q)+v \Phi N P_{\mathrm{r}}(q)\left[P_{\mathrm{r} 1 / 2}(q)-P_{\mathrm{r}}(q)\right]}{4 D(q)} \\
S_{a b}(q)=S_{b a}(q)=S^{\prime}(q)=\frac{P_{12}(q)-(v+\chi) \Phi N P_{\mathrm{r}}(q)\left[P_{\mathrm{r} 1 / 2}(q)-P_{\mathrm{r}}(q)\right]}{4 D(q)}
\end{gathered}
$$


and

$$
D(q)=\left[1-\frac{\chi}{2} \Phi N\left(P_{\mathrm{r} 1 / 2}(q)-P_{\mathrm{r}}(q)\right)\right]\left[1+\left(v+\frac{\chi}{2}\right) \Phi N P_{\mathrm{r}}(q)\right]
$$

Combining equations (15) and (16) yields:

$$
S_{\ell}(q)=\frac{4\left[P_{\mathrm{r} 1 / 2}(q)-P_{\mathrm{r}}(q)\right]}{1-\frac{\chi}{2} \Phi N\left[P_{\mathrm{r} 1 / 2}(q)-P_{\mathrm{r}}(q)\right]}
$$

\section{Discussions and conclusions.}

Figure 1 shows the variations of the form factors $P_{\ell}(q)$ (curve a), $P_{\mathrm{r}}(q)$ (curve b), and $P_{1 / 2 \mathrm{r}}(q)$ (curve $\mathrm{c}$ ) as a function of $q$. The curves a and $\mathrm{b}$ are represented by the Debye and the Casassa functions, respectively, and are found to describe quite well the neutron scattering data from dilute solutions of linear and cyclic polystyrene chains in cyclohexane [4]. One observes that, at a given value of $q$, the effect of intramolecular interferences is weaker for a half than for a total ring which in turn shows weaker interferences than a linear chain. The latter behavior can be understood since the local density of interfering monomers is higher in the case of a ring. In figure 2, we have plotted the normalized scattering intensity $4 I(q) /\left[(a-b)^{2} \Phi N\right]$ as a function of $q$ for linear (curve a) and cyclic (curve b) copolymers made of monomers of practically the same chemical nature with zero interaction parameter (i.e. $\chi=0$ ). They differ only by their scattering lengths which satisfy the condition in equation (10). The interesting remark is that the normalized intensity $4 I(q) /\left[(a-b)^{2} \Phi N\right]$ is equal to $P_{1 / 2}(q)-P_{\mathrm{r}}(q)$ independently of the polymer concentration. Similar behavior was observed in the neutron data of half deuterated polystyrene linear chains in a symmetrical mixture of ordinary and deuterated benzene [12] above the overlap concentration $c^{*}$. It would be interesting to check whether a similar observation could be made for cyclic copolymers. Obviously, this conclusion is modified when the two blocks are different and a finite parameter

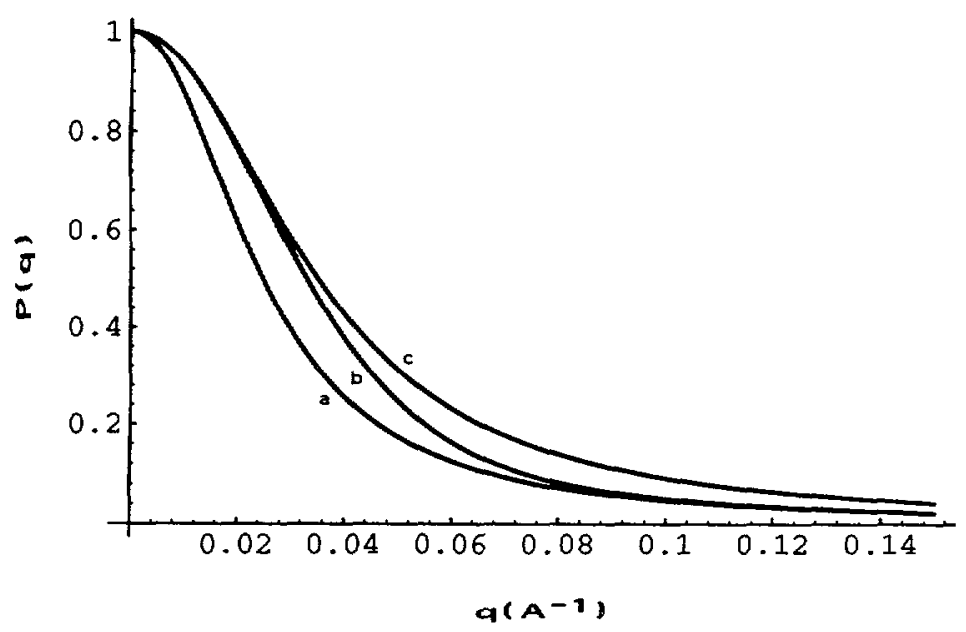

Fig. 1. - The variation of the form factors as a function of the wavevector $q$. Curve a : the case of a linear chain having the radius of gyration $R_{g}$ (i.e. Debye's function); Curve b : the case of a cyclic chain having the radius of gyration $R_{\mathrm{gr}}$ (i.e. Casassa's function); Curve c represents $P_{1 / 2 \mathrm{r}}(q)$ the form factor of one block (see Eq. (12)). 


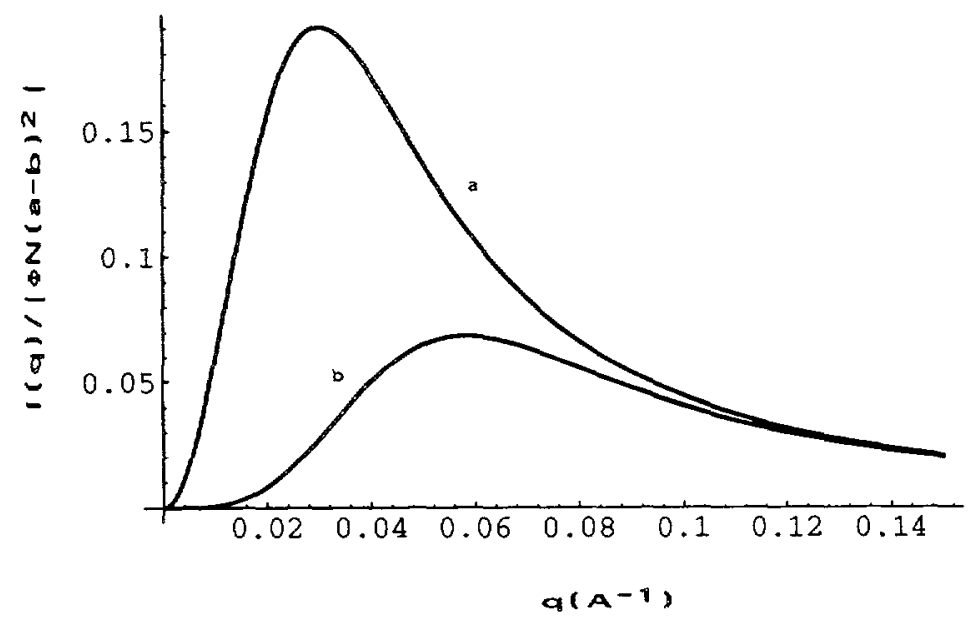

Fig. 2. - The variation of the normalized intensity as a function of the wavevector $q$ for diblock copolymers made of compatible monomers (i.e. $\chi=0$ ). Curve $a:$ linear symmetric diblock. Curve $b$ : cyclic symmetric diblock.

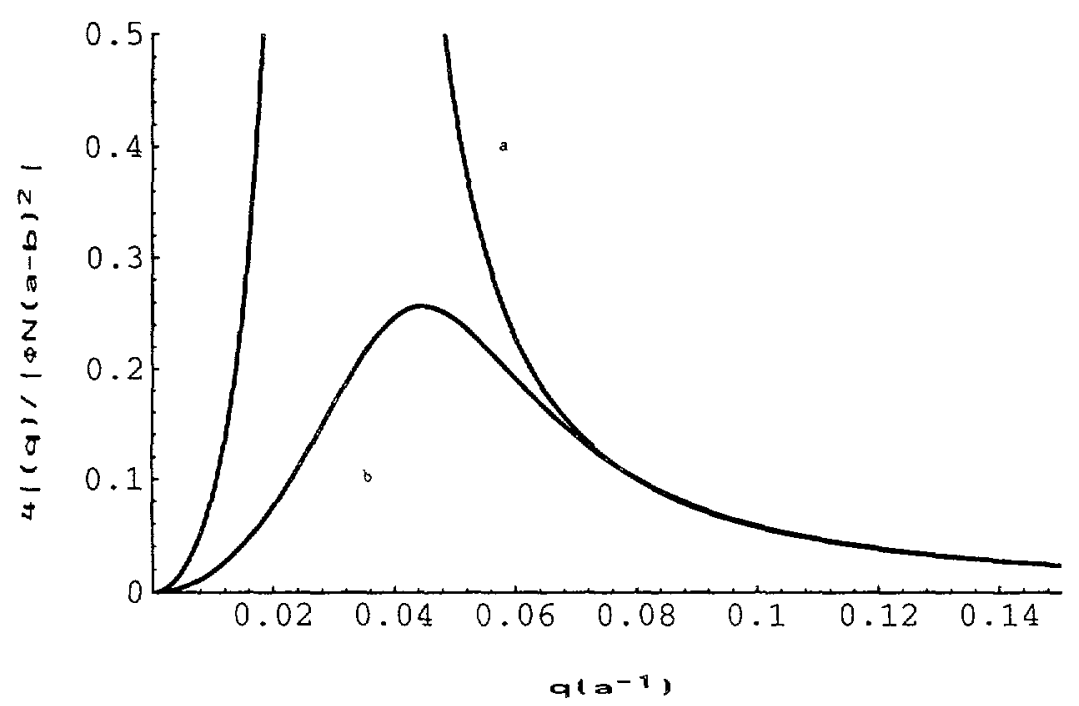

Fig. 3. - The variation of the normalized intensity as a function of the wavevector $q$ for diblock copolymers made of interacting monomers at a value of the interaction parameter in the vicinity of the MST for linear diblocks $(\chi \Phi N=10)$. Curve a : linear symmetric diblock. Curve b : cyclic symmetric diblock. The following parameters were used in these plots: $N=10^{3}$, and $\sigma=5 \AA$.

$\chi$ characterizes their incompatibility. The position of the maximum is practically not modified by $\chi$ or $\Phi$ but its height increases substantially with increasing $\chi$ or $\Phi$. In fact, if the quantity $\chi \Phi N$ reaches the value $2 /\left(P_{1 / 2 \mathrm{rm}}-P_{\mathrm{rm}}\right)$, the structure factor $S_{p}(q)$ diverges and the system undergoes an MST. Figure 3 shows the variation of the normalized intensity as a function of $q$ for $\chi \Phi N=10$ which corresponds practically to the condition of MST for the linear chains. One sees that the intensity for cyclic copolymers remains finite and comparatively small. This 
enhancement of compatibility is an essential property of mixtures involving cyclic polymers and was observed by Cates and Deutsch [16] for the first time. It is interesting to note that for the microphase transition to take place in the ring system at the concentration considered in figure 3 (and for which linear copolymers undergo an MST) one must take a critical parameter for MST $\chi_{\mathrm{mr}}$ such that $\chi_{\mathrm{mr}} \Phi N \approx 18$ which is about eighty percent higher than the corresponding value in the system of linear chains. If these results are confirmed by the experiments, it would mean that the present mean field model remains valid for cyclic copolymers much beyond its range of validity for the corresponding linear copolymers. These observations can be tested by neutron or light scattering techniques and a proper choice of the mixture to satisfy the so-called optical theta condition. In neutron scattering, one can use diblock copolymers made of ordinary and deuterated monomers in a mixture of ordinary and deuterated good solvents. The drawback of this system is that it shows a very small $\chi^{-}$ parameter. In light scattering there are several systems fulfilling the above condition and some of them were already studied in the case of linear chains [15]. Finally, one observes in figures 2 and 3 that the position of the maximum $q_{\mathrm{m}}$ is shifted to higher values indicating a shorter scale of the microstructure which may appear in the case of ring copolymers.

\section{Acknowledgements.}

We thank the referee for bringing references [2], [13], and [16] to our attention and for correcting an erroneous statement on the shift of the theta temperature for cyclic homopolymers. M. Benmouna thanks the Fondation Scientifique de Lyon (Région Rhône-Alpes) for financial support and Madame M. Rinaudo, head of CERMAV, for hospitality.

\section{References}

[1] Well R., Vingrad J., Proc. Natl. Acad. Sci. (USA) 50 (1965) 730.

[2] a) Roovers J., ToporowsKu P. M., Macromolecules 16 (1983) 843 ;

b) RoOvers J., Macromolecules 18 (1985) 1359.

[3] Zimm B. H., Stockmayer W. H., J. Chem. Phys. 17 (1946) 130.

[4] Hadzioannou G., Cotts P. M., Brinke G. ten, Han C. C., Lutz P., Strazielle C., Rempp P., Kovacs A. J., Macromolecules 20 (1987) 493, and references cited herein.

[5] Candau F., Remp P., Benoît H., Macromolecules 5 (1972) 627.

[6] Casassa E. F., J. Polym. Sci., Part A 3 (1965) 605.

[7] LEIBLER L., Macromolecules 13 (1980) 1602.

[8] de Gennes P. G., Scaling Concepts in Polymer Physics (Cornell University Press, Ithaca, 1979).

[9] Ionesco L., Picot C., Duplessix R., Duval M., Benoît H., Lingelser J. P., Gallot Y., $J$. Polym. Sci. Polym. Phys. Ed. 19 (1981) 1033.

[10] Flory P. J.. Principles of Polymer Chemistry (Cornell University Press, Ithaca, 1953).

[11] Benoît H., Wu H., Benmouna M., Bauer B., Mozer M., Lapp A., Macromolecules 20 (1987) 381 .

[12] Duval M., Picot C., Benmouna M., Benoît H., J. Phys. France 49 (1988) 1963.

[13] Benoît H., Fischer E. W., Zachmann H. G., Polymer 30 (1989) 379.

[14] Yamakawa H., Modern Theory of Polymer Solutions (Harper and Row, New York, 1971).

[15] Ould-Kaddour L., Strazielle C., Polymer 28 (1987) 459 and references cited herein.

[16] Cates M. E., Deutsch J. M., J. Phys. France 47 (1986) 2121. 